\title{
Defining the challenges - Identifying the key poisoning elements to be separated in a future integrated molten salt fast reactor clean-up system for iMAGINE
}

\author{
B. Merk ${ }^{1}$, A. Detkina ${ }^{1}$, D. Litskevich ${ }^{1}$, Michael Drury ${ }^{1}$, Omid Noorikalkhoran ${ }^{1}$, Gregory Cartland-Glover ${ }^{2}$, \\ Leon Petit ${ }^{2}$, Stefano Rolfo ${ }^{2}$, Justin P. Elliott ${ }^{3}$ and Andrew R. Mount ${ }^{3}$ \\ ${ }^{1}$ The University of Liverpool, United Kingdom \\ ${ }^{2}$ STFC Daresbury Laboratory, United Kingdom \\ ${ }^{3}$ The University of Edinburgh, United Kingdom
}

\begin{abstract}
Summary
Nuclear fission technologies have the potential to play a significant role in the energy mix of a net-zero and sustainable society. However, to achieve the sustainability goal two significant challenges remain: efficient and sustainable fuel usage and the minimisation of long term nuclear waste. Civil nuclear molten salt systems and technologies offer the opportunity to address both, delivering future reactors at scale for efficient and effective power production and nuclear waste burnup. Potentially, both objectives could be fulfilled in one reactor system, which could significantly improve sustainability indices. In addition, demand driven development of a significantly reduced fuel cycle with enhanced proliferation resistance offers further potential for improvement. To achieve these goals, a transformative approach for salt cleanup during molten salt reactor operation is proposed in this work, by concentrating on the detection and removal of key neutron poisoning elements which prevent the reactor from long-term operation. This work also demonstrates the importance of the effective integration of physics, reactor design and chemistry when systems modelling in achieving these system development goals.
\end{abstract}

\section{Introduction}

The development of nuclear fission waste reduction and management strategies has been of interest for several decades. The first strong development push was affected by two important international projects on partitioning \& transmutation (P\&T) of transuranium isotopes active in the late 1980s and early 1990s: the OMEGA (Options for Making Extra Gains from Actinides and fission products) programme in Japan and the CAPRA/CADRA programme in France. The former[1], the first major P\&T project, arose from the Japan Atomic Energy Commission's October 1988 report entitled "Long-Term Program for Research and Development on Nuclide Partitioning and Transmutation (P\&T)", which considered conversion of HighLevel Waste (HLW) into useful resources and the improvement of HLW disposal efficiency. As well as the OMEGA programme, this report plotted a course for technological development up to the year 2000. The French CAPRA/CADRA projects (CAPRA stands for Combustion Améliorée du Plutonium dans les Réacteurs Avancés or increased plutonium consumption in advanced reactors, whilst CADRA stands for Consommation d'Actinides et de Déchets dans les Réacteurs Avancés or consumption of actinides and waste in advanced reactors) were then established in 1991 by French law; this mandated a combined 15year research programme to investigate the technical options available to the nuclear fuel cycle in France [2] with the intention of investigating future options to apply fast reactors, originally foreseen for power production, to the burnup of excess plutonium, actinides and waste. Therefore, while the OMEGA project focused on partitioning as well as on transmutation, the CAPRA/CADRA project was originally only dedicated to transmutation, with a major focus of this and related reactor studies being the reuse and 
incineration of Plutonium in the Superphénix prototype fast breeder reactor. Different aspects of the potential of fast reactor systems to be used for the burning of plutonium and minor actinides (MAs) (Np, Am, $\mathrm{Cm}$ ) were considered in CAPRA and CADRA projects (Barré, 1998) ${ }^{1}$, with CAPRA mainly dealing with managing the plutonium stockpile and CADRA considering the burning (transmutation) of MAs and longlived fission products [3]. As a consequence, waste management through P\&T has mainly since been seen as a distinct task requiring a separate/special type of reactor system. More recently the potential for bringing fast reactors into operation both for wide spread power production and waste management has been explored. This has very often used a so-called double strata approach $[4,5,6]$ with sodium cooled fast reactors being proposed as the stratum (or tier) 1 system to produce energy from Plutonium and with a specific stratum/tier 2 system, e.g. an accelerator driven system (ADS) or critical solid fuelled fast reactor [5], used to efficiently burn the remaining waste (low quality plutonium and minor actinides). Now this same approach is being followed in the recently established Russian P\&T programme, but in contrast to the European programmes the Russian effort plans to use a molten salt fast reactor as its tier 2 burner system $[6,7]$. More generally, a question can now be posed: is it really necessary to have a two separated tiers approach, or would it not be possible and more conveniently go to a novel breakthrough technology, where both tiers are merged together which would in turns generate a market opportunity to deliver energy production and waste management in one integrated reactor system?

Given today's vastly different energy landscape, compared to the 1990ies when P\&T was emerging, future energy technologies have to address the urgent challenges of net zero and sustainable energy research and development, the energy trilemma and the meeting of the UN sustainable development goals (SDGs). The trilemma is characterized (e. g. by the world energy council [9] a UN-accredited body) by the three key interlinked challenges of reducing emissions, enhancing security of supply, and reducing the overall cost of energy production. The challenges of this trilemma are also reflected in the UN SDG 7: "Ensure access to affordable, reliable, sustainable and modern energy for all as one piece of sustainable development of the future world" [10]. The challenge is that waste management through P\&T can only deliver on a subset of these new requirements, while overarching objectives like enhanced/sustainable resource utilization and energy security can only be met by closed fuel cycle operation with integrated P\&T.

To meet these challenges in an integrated approach, we have proposed the iMAGINE concept, which aims at using molten salt fast reactors with integrated salt clean-up. This has the aim of operating on spent nuclear fuel and has the potential to deliver waste management [11] and power production [12] in one process. This new, integrated approach offers the prospect of a single holistic solution delivering not only an innovative system for energy production, but also a highly sustainable process e.g. through integration of the separation and transmutation processes $[13,14]$. This offers the prospect of using only one reactor system and the avoidance of the extensive fuel cycle required in the previously described double strata approach. In this work, we now address the systems development of the previously proposed iMAGINE system towards application through carrying out a feasibility study which involves the consideration and integration of physics, reactor design and systems chemistry.

\section{The iMAGINE system and its Optimization}

In the IMAGINE system, the approach to sustainable and efficient operation is to concentrate on separating out the elements which prevent the reactor system from long term operation instead of reprocessing the entire fuel. This avoids the separation of fissile and fertile material like uranium,

\footnotetext{
${ }^{1}$ Barré, B., 1998. The Future of CAPRA. 5th Int. CAPRA Seminar, Karlsruhe
} 
plutonium and the minor actinides with the consequent demand for fresh fuel production and the arising proliferation concerns. The new approach of cleaning the fuel in operation creates two further positive outcomes. First, by avoiding the proliferation issues of established reprocessing methods through avoids the separation of fissile material, it also removes the potential opportunity for misuse and theft and the consequent requirement for a massive and expensive safeguarding activity. Secondly, it has the potential to remove one of the most challenging requirements of waste management; the demand, when reprocessing and partitioning, of an extremely high separation rate of the actinides from the waste stream to avoid the loss of TRU material and its carry over into future final disposal. This problem is further aggravated in the current approach by accumulation of fissile material in the waste stream when recycling multiple times (multi-recycling) in closed fuel cycle operation and P\&T [13] To underline the serious consequences of the resulting radiotoxicity from such carry over, the influence of the separation efficiency of the TRU elements on the waste stream and the time scale of its radiotoxicity is given in Figure 1 . This observation makes it obvious that the losses result in an incomplete/insufficient result of the P\&T process, not leading to the promised sharp decrease in the radiotoxicity, but leading to a time delay of up to more than 1000 years before crossing the radiotoxicity line of the inserted fuel.

The development of the new, innovative iMAGINE approach to the separation of fission product elements will not only enable radically different thinking in transmutation, but also in nuclear and separation chemistry with the demand to deliver breakthroughs not only in molten salt reactor design and operation, but also in the development of salt clean-up processes; the iMAGINE approach will require developments in partitioning to deliver an integrated closed fuel cycle solution. 


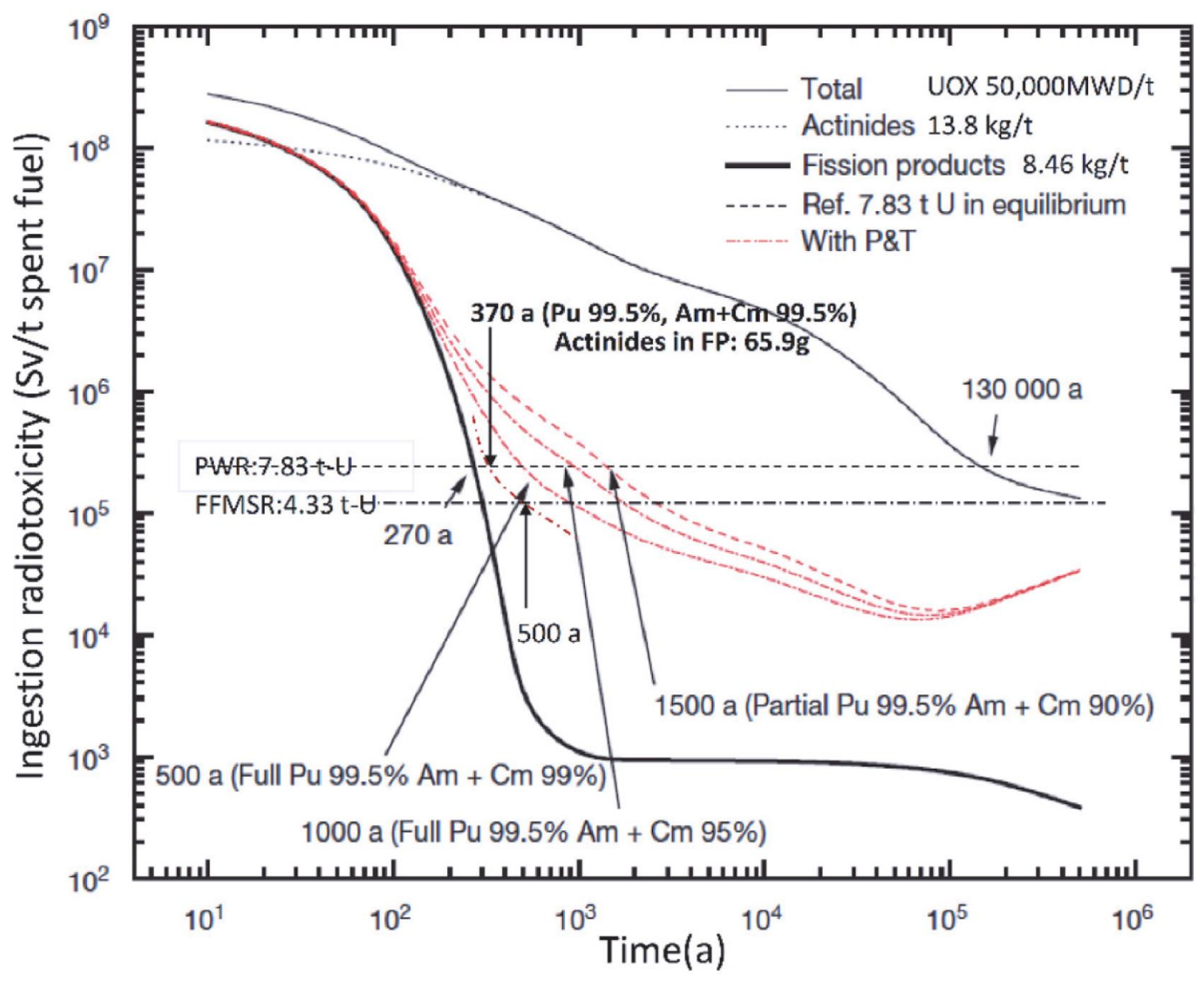

Figure 1: Radiotoxicity of nuclear waste over time and the effect of different efficiencies of the separation of plutonium and minor actinides, [16] intechopen, CC BY 3.0

In particular, the use of liquid fuelled systems, like molten salt reactors, offers the opportunity to reshape the reprocessing process completely [15] by concentrating on those elements which have the largest impact on maintaining criticality during reactor operation. This breakthrough approach would concentrate on those elemental species which through neutron adsorption have the strongest effect in reducing criticality and therefore reducing reactor fuel usage efficiency. This avoids the established approach of the complete removal/separation of fission products to regenerate the new, rigorously clean fuel required for solid fuel reactors. This new approach and these new processes offer significant optimization potential [15] around such key factors as throughput of salt through the chemical cleaning process, the selection of elements/species for separation, and the required process efficiencies of these specific separation processes. In addition, there is the potential for the significant reduction or removal of the pre- and post-processing steps required for solid fuel-based fuel cycle such as the storage and transport of the spent fuel, its cooling and dissolution, the precipitation of select materials after separation as well as the subsequent solid fuel production. Some of these steps are very costly (both economically and in terms of resources) whilst others are very time consuming and can lead potentially to significant radiation exposure of workers [7]. However, it is worth noting that these steps cannot be 
fully avoided in all fuel cycles, particularly those which rely on solid fuels and on aqueous reprocessing. In addition, there would be a further significant advantage of a process based on a fully integrated fuel cleaning system for a molten salt reactor overusing established reprocessing methods, with their demand for very high separation factors and costly fuel production and. As it would only require separation of selected fission products, the actinides would stay in the fuel system, thus potentially reducing the need for such high separation factors to achieve P\&T goals as well as avoiding the potential proliferation risk of the separated species, which is often cited as one of the highest risks of closed fuel cycle operation and P\&T.

In order to implement this attractive approach, it is important firstly to establish which elements have to be separated from the fuel to design a demand driven separation process for salt clean-up. There has already been a first attempt published [15], looking into reactor physics and identifying the elements which have the strongest influence on the system criticality, but this was based on the start-up configuration of a molten salt reactor operating on the spent nuclear fuel from light water reactors [15]. This work now delivers an additional molten salt reactor priority list, created through the modelling of the operation of a chlorine molten salt fast reactor started with enriched Uranium. The calculations for this feasibility study model use a target burnup of $100 \mathrm{GWd} / \mathrm{tHM}$.

\section{Simulation Codes \& Methods and Salt Data}

The simulations for this feasibility study were performed using fast running unstructured mesh 2D deterministic simulations based on the SCALE/POLARIS neutron transport solver. POLARIS is a module of the SCALE code system [18] for 2D deterministic transport simulation, mainly developed for cross section preparation for core simulators. POLARIS is a new module of SCALE 6.2 that provides a 2D lattice physics analysis capability, using a multigroup self-shielding method called the Embedded Self Shielding Method (ESSM) and a transport solver based on the Method of Characteristics (MoC). In general, POLARIS and its cross section library has been developed and validated for light water reactors; thus we have provided in a recent publication [19] a first verification against the Monte-Carlo code SERPENT [20] and some new, extensive follow up verifications, by comparison with different deterministic and statistic solvers of the SCALE package including some continuous energy Monte-Carlo comparisons [21, 22, 23] .

The salt data for the applied fuel composition were based on two advanced Russian publications by Katyshev and Teslyuk [24] and by Desyatnik and Katyshev [25]. The base configuration is $\mathrm{NaCl}_{-} \mathrm{UCl}_{3}-\mathrm{UCl}_{4}$ with the eutectic composition $45 \%$ (molar) $\mathrm{NaCl}-38.5 \%$ (molar) $\mathrm{UCl}_{4}-16.5 \%$ (molar) $\mathrm{UCl}_{3}$, worthy of further detailed investigation due to its relatively high loading of fertile heavy metal material at the eutectic point, which makes it a very promising composition for achieving the required breeding efficiency essential for future self-sustained breeding [19]. Thus, it is an ideal candidate to be used for a first investigation of the fission product accumulation and its effect on criticality when reaching a significant burnup to identify elements for a potential future element-based clean-up system. However, in the longer term this composition will require a large upgrading of the thermo-physical dataset to a much wider temperature range as input for any kind of future modelling and simulation studies of a potential reactor. At the mentioned eutectic point, the $\mathrm{NaCl}-\mathrm{UCl}_{3}-\mathrm{UCl}_{4}$ salt has a melting point of $338^{\circ} \mathrm{C}[24]$ and a density of $3.232 \mathrm{~g} / \mathrm{cm}^{3}$ at $980 \mathrm{~K}$.

The model for this investigation, see Figure 2, is based on and developed from two recent publications $[19,26]$. It has been developed for the evaluation of the burnup and breeding performance in a molten salt reactor as well as for the study of potential reactor control through temperature feedback. To allow 
for this, this very specific 2-D model has been built to take care of the very important effect of the neutron leakage from a molten salt reactor core. The ring type model (thin black rings indicate the grid) consists of the reactor core of variable size (red), surrounded by a stainless-steel vessel of $20 \mathrm{~mm}$ thickness (blue) and a sodium reflector of $50 \mathrm{~cm}$ thickness (yellow). The arrangement is surrounded by a thin vacuum gap (light grey rings) and the rest of the unit cell is filled with strong absorber (dark grey) to avoid the reflection of leaking neutrons from the core/reflector system through the reflective boundary conditions of the code originally developed to represent a unit cell in infinite repetition of a fuel assembly.

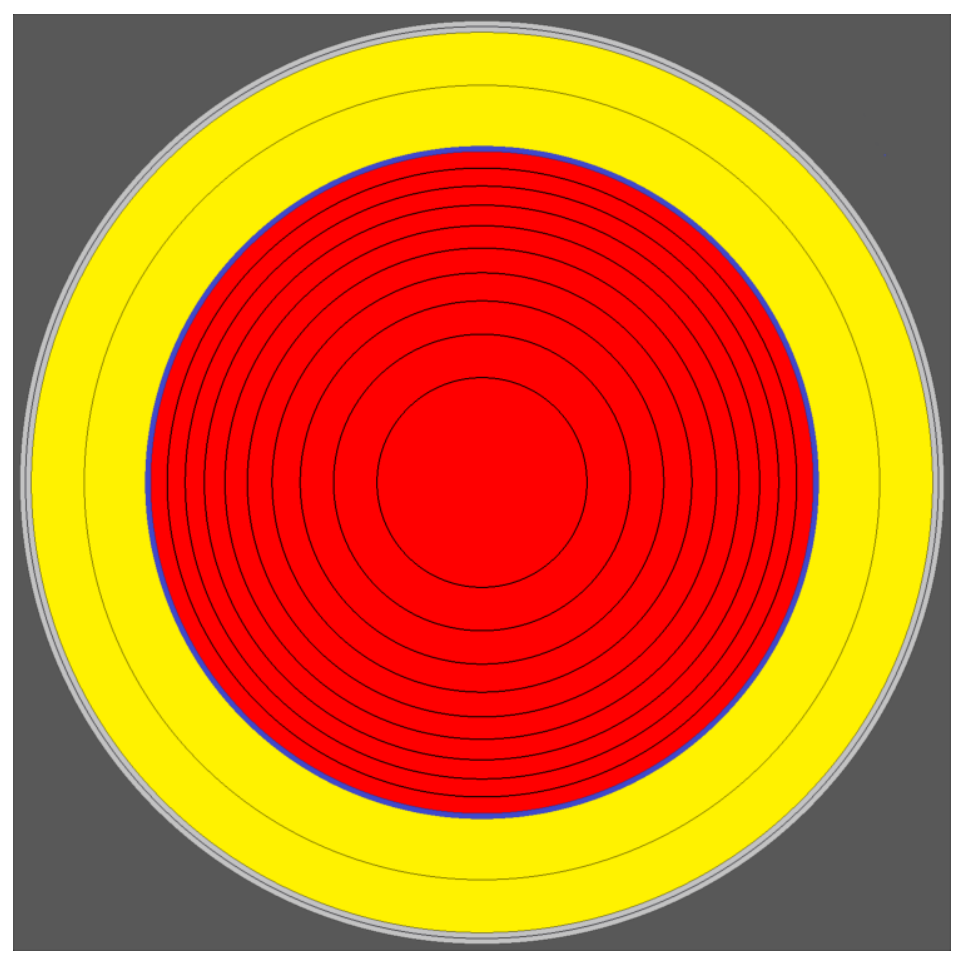

Figure 2: 2D POLARIS ring model of the full-size molten salt reactor core (red) surrounded by a steel vessel (blue), and a reflector ring (yellow), a vacuum gap (light grey), surrounded by absorber (dark grey)

\section{Modelling \& Simulation Results}

The first step was to define a reasonable base case close to eutectic composition $45 \% \mathrm{NaCl}-38.5 \% \mathrm{UCl}_{4}$ $16.5 \% \mathrm{UCl}_{3}$ which achieves a significant burnup and results in a sufficient amount of fission products to create sufficient effect on criticality by each fission product. As well as being important to get a reliable estimation of the effect each fission product element has on criticality, it is also required to get insight into the likely concentrations of fission products as a basis for the design of a future separation (cleanup) system. The analysis is based on the standard case of [26], with a system radius of $213 \mathrm{~cm}$, a target burnup of $100 \mathrm{GWd} / \mathrm{tHM}$, an initial U-235 enrichment of 11.78 at\% which has been found in an iterative approach. The system size was iterated in a multi-stage approach. The first iteration is used to determine an enrichment which delivers an initial criticality for each size within an accuracy of $<10 \mathrm{pcm}$, while in the second stage a burnup case was run to determine the maximum criticality achieved through 
the burnup with the aim of determining the radius that delivers a maximum $k_{\text {eff }}$ of 1.006 at the point of highest criticality, see Figure 3.

The used model shows a typical burnup behaviour of a breeder system (see Figure 3, or [19]) which is characterized by a profile starting with a growth in criticality until a single, central peak followed by a continuous decrease. The curve starts at criticality (iteration condition) with the degree of criticality rising initially caused by the breeding of new fissile material from the fertile U-238, see Figure 5 . In general, the breeding and burnup behaviour of a reactor core is characterized by a competition between different processes: a) the formation of new fissile material, b) the consumption of fissile and fertile material, and c) the progressive build-up of fission products which has an increasingly strong and long term effect. Thus, the first part of the curve is dominated by breeding, which delivers an increase in criticality, whilst the second part is dominated by fission product accumulation, see Figure 6 (and the slow decrease in the amount of fertile U-238 which slightly slows the breeding) which deliver a progressive decrease in criticality.

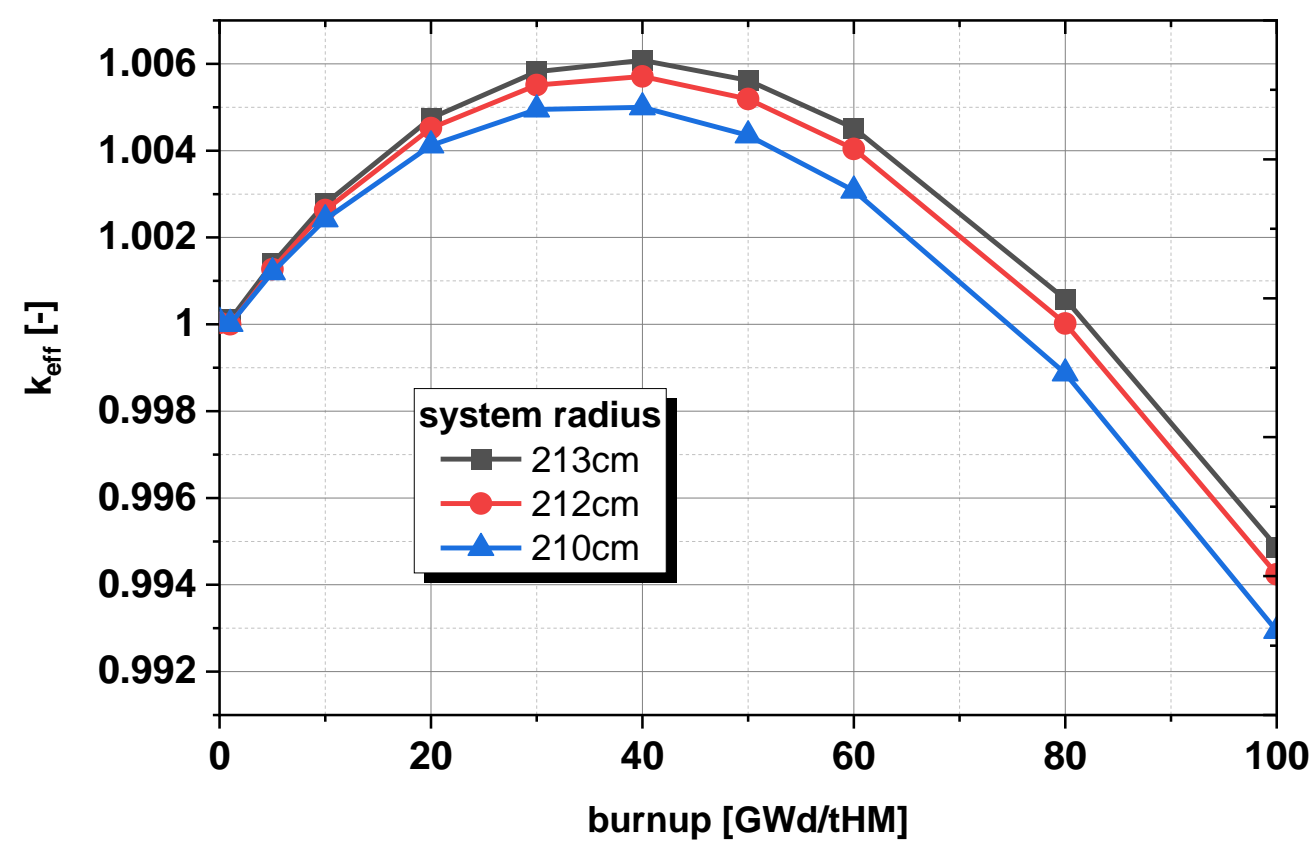

Figure 3: Criticality of the 2D finite system, as measured by $k_{\text {eff, }}$ as a function of burnup (time) for different system radii for the close to eutectic composition $45 \% \mathrm{NaCl}-38.5 \% \mathrm{UCl}_{4}-16.5 \% \mathrm{UCl}_{3}$

An extended burnup curve is given in Figure 4, with the reference (base) case radius $213 \mathrm{~cm}$ again showing the initial increase in criticality caused by breeding and the longer-term reduction of the criticality due primarily to fission product accumulation as given in the black curve of Figure 3 . To identify the potential maximal effect of a clean-up system which would remove all fission products, the 
criticality development of a theoretical system without fission product accumulation is then given for comparison. This result demonstrates both the large breeding which takes place in this system (see the $\mathrm{Pu}$ accumulation in Figure 5) and the tremendous effect of the fission product accumulation on system criticality (see Figure 6). The general form of the breeding curve is comparable to results for other breeding based systems like sodium cooled fast reactors [27], while the detailed result is dominated by the core size/leakage and the correlation between fertile and fissile load in the system.

The detailed Pu breeding (Figure 5) shows the growth of the plutonium content from zero in the initial composition to almost $10000 \mathrm{ppm}$ at the final burnup composition. Long term all Pu isotopes shows an asymptotic approach to a steady-state value characterized by the specific balance between the breeding and the fission processes. The plutonium vector is mainly dominated by Pu-239. The higher Pu isotopes take longer to form but long-term, Pu-240 shows an increased steady-state amount due to its lower fission cross section whilst Pu-241, with its significantly higher fission cross section, has a lower steadystate amount.

The fission product accumulation, Figure 6 is seen to be almost linear with burnup for 5 leading fission product elements. In general, it is not surprising that the amount of fission products formed is directly proportional to the number of fission events which have taken place and thus to the amount of energy produced in the system (the burnup). Thinking a bit further into the future, this behaviour would change to asymptotic behaviour, with element concentration plateauing at a steady state value, if some constant share of the specific fission product element could be removed by a salt clean-up system.

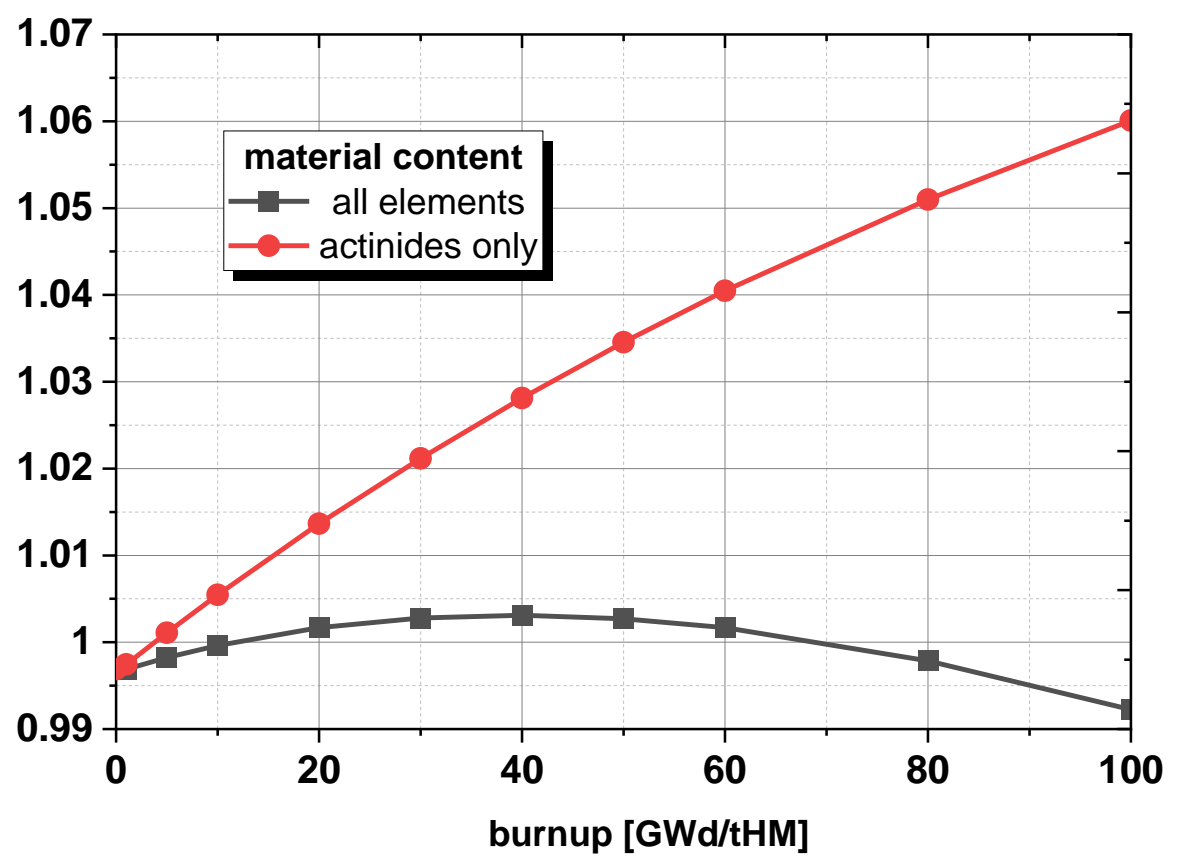

Figure 4: Criticality over burnup for the base case $(213 \mathrm{~cm}$ from Fig. 2) for the investigation compared to criticality development for a virtual system without fission products 


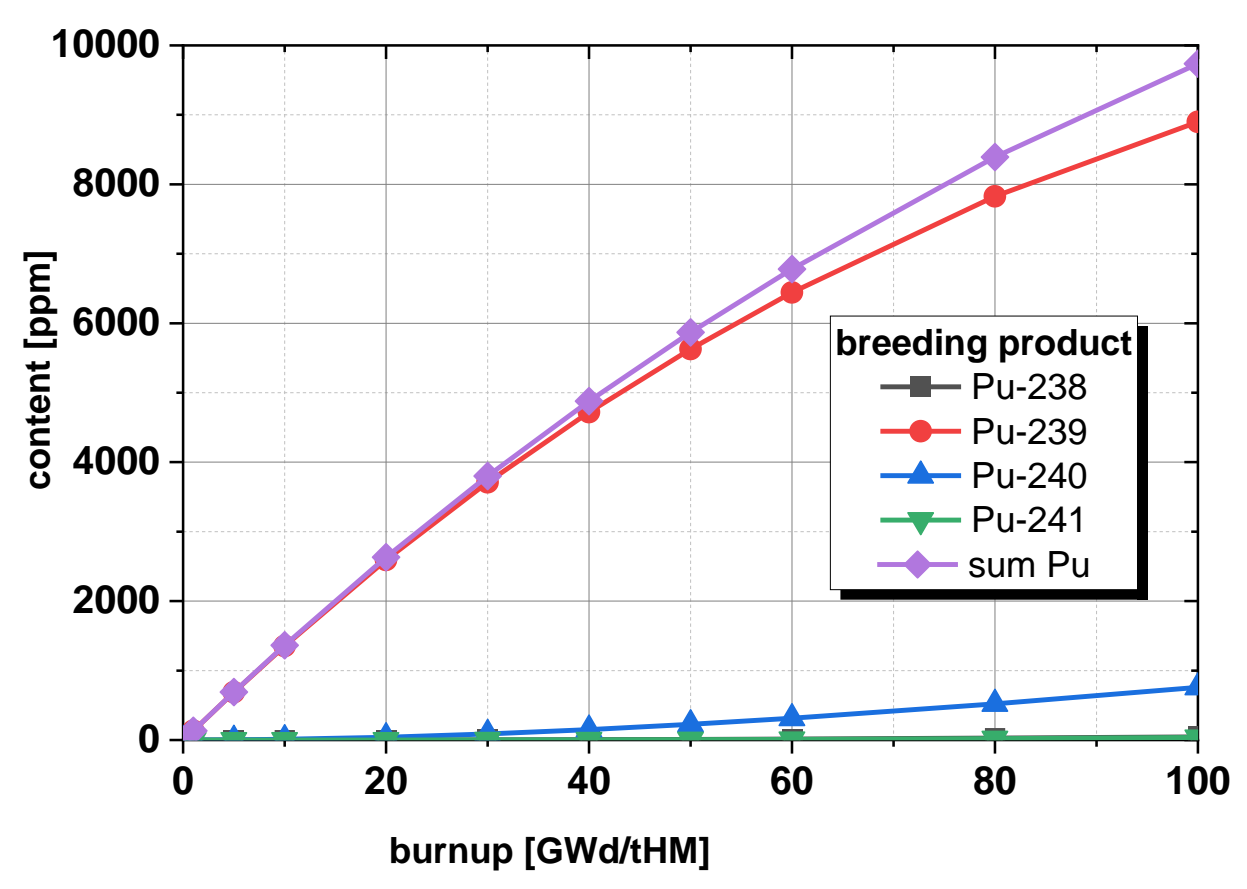

Figure 5: Breeding product accumulation over burnup for selected leading breeding products

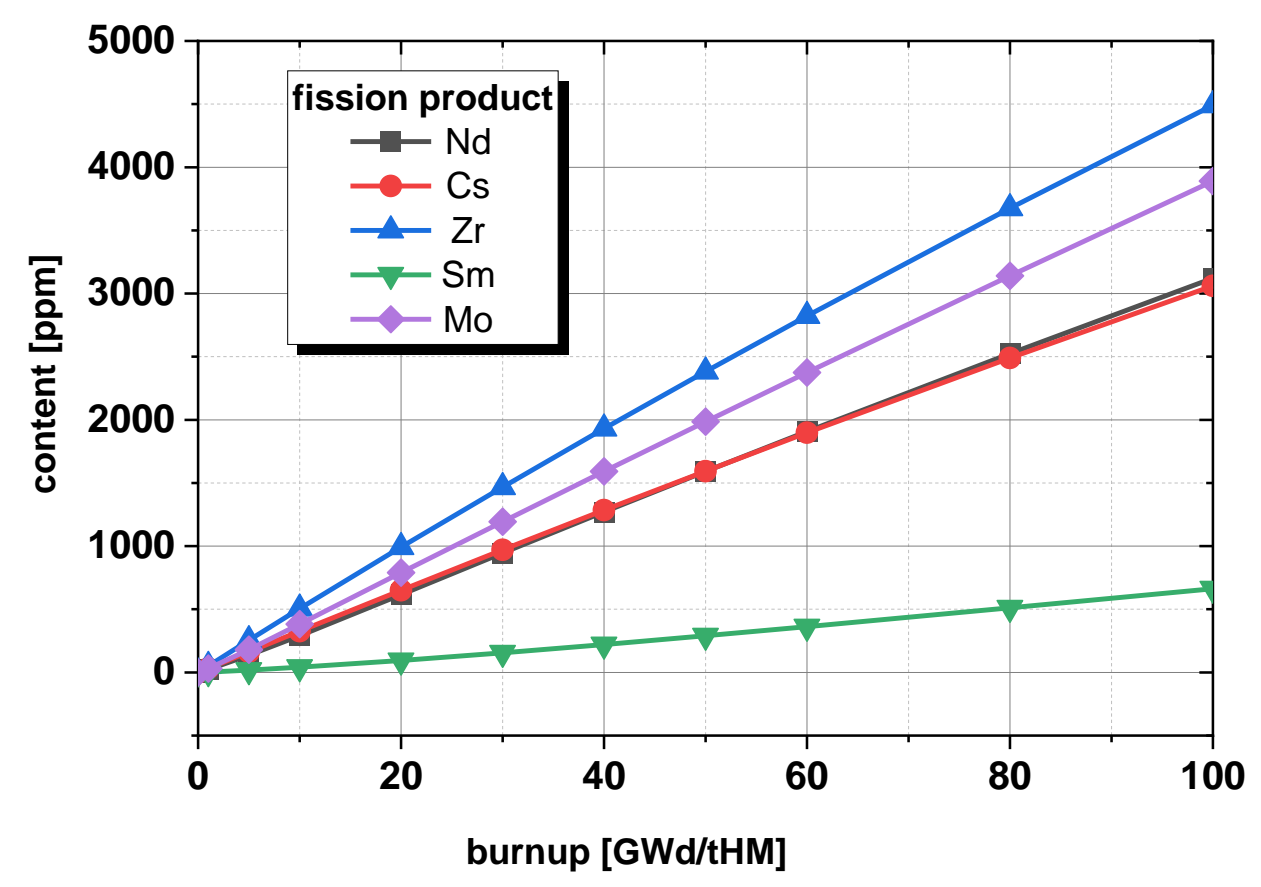

Figure 6: Fission product accumulation over burnup for selected leading fission products 
For the full investigation of fission product accumulation, the 142 isotopes of highest concentration were evaluated from the POLARIS calculation as tested in [28]. The accumulation of these isotopes was analysed in detail and the leading isotopes of up to 37 elements have been considered.

The criticality effect of each of the different elements was determined through use of a very specific procedure which has been developed and tested recently in [15]. At the target burnup of $100 \mathrm{GWd} / \mathrm{tHM}$ the detailed composition was reproduced as a new starting input for a follow up calculation. This base case including all fission products indicated a criticality value of $\mathrm{k}_{\mathrm{ref}}=0.99224$, used as the reference value. Based on this input a series of steady state calculations was then initiated to study the effect of the removal of the different fission product elements. Each time an element $\mathrm{x}$ was removed and the calculation repeated, based on the new criticality value, $\mathrm{k}_{\mathrm{x}}, \Delta \mathrm{k}_{\text {eff }}$ in $\mathrm{pcm}$ was generated using equation 1.

$\Delta k_{e f f}[p c m]=\frac{k_{x}-k_{r e f}}{k_{r e f} \cdot k_{x}} \cdot 10^{5}$

All major elements which result in a more than $50 \mathrm{pcm}$ criticality effect (change in keff), are given in Table 1. The comprehensiveness of the chosen isotope and element set can be observed in the last two lines of the table. The line sum indicates the summed values of all considered isotopes in the table while the line whole indicates the effects when the whole isotope set of the POLARIS code is used. Thus, the considered isotope and element set given in the table considered $93 \%$ of the overall fission product concentration and $95 \%$ of the criticality reduction due to the fission product accumulation. The concentrations of these leading elements, in terms of their poisoning effect, can be seen to vary from Zirconium (at almost 4500 ppm) to Europium ( 50 ppm) which reflects the widely differing neutron capture cross sections of these isotopes. One approach, in terms of developing potential separation methods, could be to only consider those elements with a significant concentration (say more than 500 ppm). This reduces the number of elements from 20 to 16 . However, the ultimate decision criterion as which to elements should be separated should be those with the highest effect on criticality (combined through the content and the neutron capture cross section), which is readily assessed through $\Delta \mathrm{k}_{\text {eff. }}$ This indicates Ruthenium and Molybdenum as elements with the highest influence on criticality with more than $700 \mathrm{pcm}$ effect after $100 \mathrm{GWd} / \mathrm{tHM}$ of burnup at reasonably high concentrations of 2500 to almost 4000 ppm. The next elements are Caesium, Neodymium, and Palladium with a criticality effect between 500 and $600 \mathrm{pcm}$, with both $\mathrm{Nd}$ and Cs each having a reasonable concentration of more than $3000 \mathrm{ppm}$, while the concentration of Palladium is only about 1000 ppm. The next level would be Technetium and Samarium with a criticality effect of slightly below $500 \mathrm{pcm}$, but both elements have a relatively low concentrations below 1000 ppm. The strong relative effect of the Europium is highlighted in addition. This element has by far the strongest effect on criticality per unit mass, as a result of the high capture cross section. However, the challenge of separating Europium would be high due to its relatively low concentration.

The overall criticality effect of all evaluated elements accounts to $6478 \mathrm{pcm}$ in criticality loss, which correlates well with the result obtained without considering the fission products, see Figure 4, which gives a criticality difference of $6448 \mathrm{pcm}$. Thus, both approaches deliver results which agree within the 
calculation accuracy expectations. In addition, it has to be mentioned that in the current criticality loss calculations all gaseous fission products are still considered as dissolved, thus the real criticality loss of a molten salt reactor would be lower due to release of at least a part of the gaseous fission products (e. $\mathrm{g}$. $\mathrm{Xe}$ ) and fission products (like $\mathrm{I}_{2}$ ) with low melting points which will volatilise.

Table 1 : Concentration of the leading fission product poison elements accumulated after a burnup of $100 \mathrm{GWd} / \mathrm{tHM}$ assessed through their correlated influence on the system criticality

\begin{tabular}{|c|c|c|c|c|c|c|c|}
\hline Element & $\begin{array}{c}\text { Concentration } \\
{[\mathrm{ppm}]}\end{array}$ & $\begin{array}{c}\text { Conc. } \\
\text { Percentage } \\
\text { Fraction [\%] }\end{array}$ & $\begin{array}{c}\Delta \mathrm{k}_{\text {eff }} \\
{[\mathrm{pcm}]}\end{array}$ & $\begin{array}{c}\Delta \mathrm{k}_{\text {eff }} \\
\text { Percentage } \\
\text { Fraction [\%] }\end{array}$ & $\begin{array}{c}\Delta \mathrm{k}_{\text {eff }} / \\
\text { Concentration } \\
{[\mathrm{pcm} / \mathrm{ppm}]}\end{array}$ & \multicolumn{2}{|c|}{$\begin{array}{c}\text { Range of } \\
\text { Oxidation } \\
\text { States }\end{array}$} \\
\cline { 5 - 8 } & & & & & & Min & Max \\
\hline $\mathrm{Eu}$ & 53 & 0.17 & 80 & 1.30 & 1.509 & $2+$ & $3+$ \\
\hline $\mathrm{Pm}$ & 89 & 0.28 & 69 & 1.12 & 0.775 & $3+$ & $3+$ \\
\hline $\mathrm{Sm}$ & 663 & 2.08 & 491 & 7.97 & 0.741 & $2+$ & $3+$ \\
\hline $\mathrm{Tc}$ & 894 & 2.81 & 493 & 8.00 & 0.551 & $3+$ & $4+$ \\
\hline $\mathrm{Rh}$ & 696 & 2.19 & 324 & 5.26 & 0.466 & $3+$ & $3+$ \\
\hline $\mathrm{Pd}$ & 1139 & 3.58 & 503 & 8.16 & 0.442 & $2+$ & $2+$ \\
\hline $\mathrm{H}$ & 647 & 2.03 & 251 & 4.07 & 0.388 & $1+$ & $1+$ \\
\hline $\mathrm{I}$ & 179 & 0.56 & 62 & 1.01 & 0.346 & $1-$ & $0-$ \\
\hline $\mathrm{Ru}$ & 2512 & 7.90 & 739 & 11.99 & 0.294 & $2+$ & $3+$ \\
\hline $\mathrm{Cs}$ & 3061 & 9.62 & 603 & 9.78 & 0.197 & $1+$ & $1+$ \\
\hline $\mathrm{Mo}$ & 3890 & 12.23 & 725 & 11.76 & 0.186 & $2+$ & $6+$ \\
\hline $\mathrm{Nd}$ & 3122 & 9.81 & 569 & 9.23 & 0.182 & $2+$ & $3+$ \\
\hline $\mathrm{Pr}$ & 904 & 2.84 & 122 & 1.98 & 0.135 & $3+$ & $3+$ \\
\hline $\mathrm{Rb}$ & 471 & 1.48 & 52 & 0.84 & 0.110 & $1+$ & $1+$ \\
\hline $\mathrm{Xe}$ & 3685 & 11.58 & 346 & 5.61 & 0.094 & 0 & 0 \\
\hline $\mathrm{La}$ & 999 & 3.14 & 88 & 1.43 & 0.088 & $3+$ & $3+$ \\
\hline $\mathrm{Zr}$ & 4491 & 14.12 & 364 & 5.91 & 0.081 & $3+$ & $4+$ \\
\hline $\mathrm{Ce}$ & 1944 & 6.11 & 135 & 2.19 & 0.069 & $3+$ & $4+$ \\
\hline $\mathrm{Ba}$ & 1309 & 4.11 & 89 & 1.44 & 0.068 & $2+$ & $2+$ \\
\hline $\mathrm{Sr}$ & 1067 & 3.35 & 58 & 0.94 & 0.054 & $2+$ & $2+$ \\
\hline $\mathrm{sum}$ & 31815 & 100 & 6163 & 100 & & & \\
\hline $\mathrm{whole}$ & 34179 & & 6478 & & 0.190 & & \\
\hline $\mathrm{Th}$ & & & & & & & \\
\hline
\end{tabular}

The results given in Table 1 now form a basis for discussion of the interplay between reactor physics and chemistry. It should be noted that to date simulation results have been delivered through the use of a lattice code which is optimized solely for the determination of criticality, and which results in the highlighting of those major isotopes with most effect on criticality through $\Delta \mathrm{k}_{\text {eff. }}$. However, this only returns those elements whose removal has the largest effect on criticality, without consideration of the 
ease of their removal chemistry. This requires additional consideration of their chemical properties in terms of the range of oxidation state(s) possible in the molten salt (Table 1), the resulting speciation (chemical environment) which may be burnup dependent and the process(es) by which they may then be separated. Building in such considerations may require a wider set of fission products to be considered, more than the criticality relevant fission products which are typically followed in a lattice code. Therefore, as a next step for this wider consideration it is advisable to recheck this given list with an inventory code like, ORIGIN or FISPIN, to assure that a complete set of fission products is considered. This would then lead to more comprehensive studies which aim to model or understand this chemical speciation, any chemical reactions and the resulting chemical and physical properties. Using an inventory code will then inform the best possible approaches for future salt clean-up based on the chemical nature and environments of the different elements. One simple example would be lodine, for which formation in the -1 oxidation state should result in retention in the molten salt and potential complex formation, whilst formation in zero oxidation state as $I_{2}$ should result in ready volatilisation without the need for chemical separation.

Therefore, although initial inspection of Table 1 suggests the development of separation technologies for Ruthenium and Molybdenum should be prioritised for salt clean-up, due to their preferential formation during burnup and their highest adverse effect on criticality, practical considerations such as feasibility, economics, viability at scale and the separation rates of separation technologies also need to be considered. Ultimately, the final decision of which elements to separate from the fuel salt can only be made after evaluation of the possible separation technologies available for the elements present in their oxidation state(s). This is challenging, but opens up a new optimization opportunity within this work.

A further challenge in molten salt reactor operation will also be in the control and monitoring of the physical and chemical state of the reactor and the associated clean up processes. One application will be in salt clean-up and its feedback into reactor operation. This is discussed in [15] in terms of the new optimization approaches made possible by integrated molten salt reactor systems, for which no pre- and post-processing of the fuel is required, as distinct from current technologies which use solid fuelled reactors and aqueous reprocessing. Another application will be in operational sensing and monitoring, which will be one of the most essential components of molten salt reactor systems in the short as well as the long term. This is because the fission process will result in a continuous evolution of the composition and potentially the chemical and physical properties of the salt through breeding, the fission process itself and fission product formation. Thus, monitoring and specific adjustment of this salt composition will likely be required for stable operation in addition to extraction for clean-up. A key challenge will be to keep the thermochemical, thermodynamic and redox environments as stable as possible for reliable operation. One example of the consequences of fission on reactor redox chemistry is the fission of one Uranium atom present as a U(IV) species in chloride, which results in $\mathrm{Ba}(\mathrm{II})$ and $\operatorname{Kr}(0)$ and the oxidation of 2 chloride ligands to produce free chlorine gas and/or the further conversion of $\mathrm{U}(\mathrm{III})$ to $\mathrm{U}(\mathrm{IV})$. Without control of such reactions, operation could lead to significant deviation from the eutectic composition of $45 \% \mathrm{NaCl}-17 \% \mathrm{UCl}_{3}-38 \% \mathrm{UCl}_{4}$, with the consequent change in the thermo-physical and thermochemical properties of the molten salt. A worst-case scenario could include dramatic 
changes in heat transfer and fluid dynamic properties induced by solidification or segregation, as a result of the consequent increase in melting point of the salt when deviating from the eutectic. Another aspect where coupled chemical consideration is required is in fission product solubility and release of gaseous fission products (like the noble gases). As well as for all fission products, which could be released in the form of aerosols due to the high temperature of the molten salt pool. This can be relevant since the melting point of the eutectic is much lower than for other compositions, which means in the case the reactor is not operated at the eutectic, higher temperatures will be required. This just demonstrates the importance of close inter-disciplinary interactions and coupled chemical and physical considerations for successful design and operation of such a molten salt reactor system. The chlorine balance alone will require a coupled modelling and simulation campaign alone, delivering fission product composition as well as the number of fissions for reactor physical models into the chemistry model and its feedback form chemistry to physics, e. g. due to solubility and release effect for gaseous fission products. A molten salt reactor system like iMAGINE, operating on a closed fuel cycle on spent nuclear fuel without prior reprocessing should be seen as one approach to integrate a whole fuel without the requirement for storage, transport or external solid fuel production. However, this approach will still require further essential chemical steps directly linked to reactor operation; the separation of fission products, their immobilization and the handling and control of the salt phase.

\section{Conclusions and Outlook}

Molten salt reactors such as the proposed IMAGINE system provide new opportunities for large energy production as well as for nuclear waste management in an integrated system. Ideally, this technology development will help to link these two objectives effectively for the first time since industrial energy production in fast reactors ceased in western countries. Molten salt reactor systems offer this opportunity due to a liquid phase core and absence of the requirement for pre- and post- processing of the fissile material compared to alternative classical solid fuelled reactor systems.

There is a clear opportunity and demand for a new type of reprocessing, which separates off those elements that reduce the efficiency of the neutron economy, thereby preventing long term reactor operation, in contrast to the established current approach of separating fissile and fertile materials. This new approach will deliver two major advantages; it will help to avoid proliferation concerns due to the absence of separated fissile material and it will avoid a so-called 'plutonium economy'. This is due to Pu staying inside the reactor system at all times. Here, the Pu acts as a kind of catalyst enabling the use of $\mathrm{U}-238$ as an almost unlimited energy resource through a breed and burn system, which should create a factor of up to 100 times more energy out of the given Uranium resource. This seems an attractive alternative to expensive and proliferation enhancing separation and reinsertion steps in the closed fuel cycle, as well as to solid fuel fabrication. The proposed new approach also could improves financial sustainability since it eliminates most of the costly and complex fuel cycle steps required in other reactor systems . Furthermore, this proposed, innovative approach could further reduce costs and complexity through avoiding the traditional requirements of extremely high separation rates and efficiencies for classical reprocessing. These demanding traditional requirements are a consequence of 
the impact of any residual TRUs into the final disposal stream with consequent implications for the waste management strategy and the required observation and active cooling times required in final disposal.

For this new, innovative approach an important first step outlined here is to identify the most harmful elements which poison the neutron chain reaction and effect core criticality, since these are the elements for which the separation processes are likely to be most effective. A series of calculations of the amounts of the specific elements appearing in the core after a burnup of $100 \mathrm{GWd} / \mathrm{tHM}$ have identified Ruthenium and Molybdenum as elements with the highest influence on criticality, each formed after $100 \mathrm{GWd} / \mathrm{tHM}$ burnup at the reasonably high concentration of 2500 to almost $4000 \mathrm{ppm}$. The next elements identified were Caesium, Neodymium, and Palladium.

This work is an important first step; however, to be really successful in not only revolutionizing the reactor design, but also in creating effective fuel cycle integration, it is essential to create a much closer link between reactor physics, reactor design and operation, reactor chemistry and the chemistry of processing. Additional challenges which result include the requirement for a coupled modelling \& simulation strategy which deals with changes in the chemical composition of the salt during burnup and the resulting effects on the physicochemical properties. For example, changes in chemical composition could lead to changes in physical properties, redox states and e.g. the amount of free and bound chloride in the salt, which need to be monitored and potentially addressed through selected chemical addition as well as targeted chemical extraction in such a system.

The modelling of all relevant physical and chemical processes and their potential interactions, including reactor operation, salt clean-up, and change in the salt composition is therefore required, which will necessitate an integrated, interlinked and dynamic procedure for modelling of the complete reactor processes.

\section{Acknowledgement}

This project was sponsored through the STFC Research Grant «Depositio-MSFR: a numerical study for the surface plating of noble metals in Molten Salt Fast Reactors (ST/W002310/1)» under the STFC Horizons Programme Solutions to Net Zero call.

\section{References}

1. Nakamura M et al. (1992) Present Status of the Omega Program in Japan. Second International Information Exchange Meeting on Actinide and Fission Product Separation and Transmutation Argonne, USA, 1992, https://www.oecd-nea.org/pt/docs/iem/argonne92/arg02.pdf, accessed 10.06.2013

2. Plutonium Management in the Medium Term A Review by the OECD/NEA Working Party on the Physics of Plutonium Fuels and Innovative Fuel Cycles (WPPR), Nuclear Science ISBN 92-64-02151-5, OECD 2003, http://www.oecd-nea.org/science/docs/pubs/nea4451-plutonium.pdf, accessed 10.06.2013 
3. Vasile A et al. (2001)The Capra - Cadra Programme", Proc. ICONE 8, 8th conference on Nuclear Engineering, April 2-6 2000, Baltimore,US, Professional Engineering Publishing, Suffolk, UK.

4. H.Takano, K.Nishihara, K.Tsujimoto, T.Sasa, H.Oigawa, T.Takizuka (2000) Transmutation of long-lived radioactive waste based on double-strata concept, Progress in Nuclear Energy, Volume 37, Issues 14, 2000, Pages 371-376, https://doi.org/10.1016/S0149-1970(00)00073-1

5. M. Salvatores ADVANCED OPTIONS FOR TRANSMUTATION STRATEGIES, avaiable: https://core.ac.uk/download/pdf/21748160.pdf, accessed 08/04/2020

6. E.M.González-Romero (2011) Impact of partitioning and transmutation on the high level waste management, Nuclear Engineering and Design, Volume 241, Issue 9, September 2011, Pages 34363444

7. A. Khaperskaya: Conceptual approaches and the main directions of $R \& D$ on partitioning and transmutation of minor actinides and long-lived fission products in the Russian Federation 15th IEM P\&T, 30 Sept.3 Oct. 2018, Manchester Hall, Manchester, UK

8. https://www.sibghk.ru/news/9068-na-gkhk-proshlo-rabochee-soveshchanie-po-voprosu-sozdaniyazhidkosolevogo-reaktora.html

9. https://www.worldenergy.org/work-programme/strategic-insight/assessment-of-energy-climatechange-policy/

10. https://unchronicle.un.org/article/goal-7-ensure-access-affordable-reliable-sustainable-and-modernenergy-all

11. B. Merk, D. Litskevich, M. Bankhead, R. Taylor (2017), "An innovative way of thinking Nuclear Waste Management - Neutron physics of a reactor directly operating on SNF", PLOS ONE July 27, 2017, https://doi.org/10.1371/journal.pone.0180703

12. B. Merk, D. Litskevich, K. R. Whittle, M. Bankhead, R. Taylor, D. Mathers (2017), "On a Long Term Strategy for the Success of Nuclear Power", ENERGIES, 8(11), 12557-12572. doi:10.3390/en81112328.

13. B. Merk et al. (2019),"The Current Status of Partitioning \& Transmutation and how to develop an Innovative Approach to Nuclear Waste Management", atw 5

14. B. Merk et al (2019) "iMAGINE - A disruptive change to nuclear or how can we make more out of the existing spent nuclear fuel and what has to be done to make it possible in the UK?", atw 6/7

15. Magill, J. \& Berthou, V. \& Haas, D. \& Galy, J. \& Schenkel, R. \& Wiese, H.-W \& Heusener, G. \& Tommasi, J. \& Youinou, G.. (2003). Impact Limits of Partitioning and Transmutation Scenarios on Radiotoxicity of Actinides in Radioactive Waste. Nuclear Energy-journal of The British Nuclear Energy Society - NUCL ENERG-J BRIT NUCL ENERG. 42. 263-277. 10.1680/nuen.42.5.263.37622.

16. Y. Hirose, Fast-Spectrum Fluoride Molten Salt Reactor (FFMSR) with Ultimately Reduced Radiotoxicity of Nuclear Wastes DOI: 10.5772/intechopen.90939

17. B Merk, D Litskevich, R Gregg, AR Mount (2018), « Demand driven salt clean-up in a molten salt fast reactor-Defining a priority list », PloS one 13 (3), e0192020

18. M. A. Jessee, J. J. Jarrell, W. A. Wieselquist, M. L. Williams, K. S. Kim, T. M. Evans, S. P. Hamilton, C. A. Gentry (2017), "POLARIS - 2D LIGHT WATER REACTOR LATTICE PHYSICS MODULE, in SCALE Code System”, edited by B.T. Rearden, M.A. Jessee, February 2017

19. B. Merk, A. Detkina, S. Atkinson, D. Litskevich, G. Catland-Glover: Evaluation of the Breeding Performance of a NaCl-UCl-Based Reactor System, Energies 2019, 12(20), 3853; https://doi.org/10.3390/en12203853

20. J. LEPPÄNEN et. al. (2015), "The Serpent Monte Carlo code: Status, development and applications in 2013." Ann. Nucl. Energy, 82 142-150.

21. Merk, B.; Detkina, A.; Atkinson, S.; Litskevich, D.; Cartland-Glover, G. On the Dimensions Required for a Molten Salt Zero Power Reactor Operating on Chloride Salts. Appl. Sci. 2021, 11, 6673. https://doi.org/10.3390/app11156673 
22. Merk, B.; Detkina, A.; Atkinson, S.; Litskevich, D.; Cartland-Glover, G. Evaluating Reactivity Control Options for a Chloride Salt-Based Molten Salt Zero-Power Reactor. Appl. Sci. 2021, 11, 7447. https://doi.org/10.3390/app11167447

23. Merk, B.; Detkina, A.; Atkinson, S.; Litskevich, D.; Cartland-Glover, G. Innovative Investigation of Reflector Options for the Control of a Chloride-Based Molten Salt Zero-Power Reactor. Appl. Sci. 2021, 11, 6795. https://doi.org/10.3390/app11156795

24. Katyshev, S. , Teslyuk, L (2018)," Ionic Melts in Nuclear Power", in Challenges and Solutions in the Russian Energy Sector, Springer International Publishing

25. Desyatnik, V.N., \& Katyshev, S.F. (1980). Volumetric and surface properties of the $\mathrm{NaCl}_{-} \mathrm{UCl}_{3}-\mathrm{UCl}_{4} \mathrm{melts}_{\text {. }}$ Zhurnal Fizicheskoj Khimii, 54(6), 1606-1610.

26. Merk, B.; Detkina, A.; Litskevich, D.; Atkinson, S.; Cartland-Glover, G. The Interplay between Breeding and Thermal Feedback in a Molten Chlorine Fast Reactor. Energies 2020, 13, 1609.

27. Merk, B., Devan, K., Bachchan, A., Paul, D., Puthiyavinayagam, P., \& Srinivasan, G. (2017). Can enhanced feedback effects and improved breeding coincide in a metal fueled, sodium cooled fast reactor?. ANNALS OF NUCLEAR ENERGY, 105, 205-218.

28. B. Merk, A. Detkina, S. Atkinson, D. Litskevich, G. Cartland-Glover: Evaluation of the Breeding Performance of a NaCl-UCl-Based Reactor System, Energies 2019, 12(20), 3853; https://doi.org/10.3390/en12203853 